\title{
Conditions for division and multiplication of frequencies in ferrite
}

\author{
Dmitriy Pleshev ${ }^{1,2 *}$, Vladimir Vlasov ${ }^{2}$, Fanur Asadullin ${ }^{1,2}$, Leonid Kotov ${ }^{2}$, Sergey Poleshchikov ${ }^{1}$, Vladimir Shavrov ${ }^{3}$, and \\ Vladimir Shcheglov ${ }^{3}$
}

${ }^{1}$ Saint-Petersburg state forest technical university named after S.M. Kirov, St-Petersburg, Russia

${ }^{2}$ Syktyvkar State University, Syktyvkar, Russia

${ }^{3}$ Institute of Radioengineering and Electronics of the Russian Academy of Sciences, Moscow, Russia

\begin{abstract}
The paper considers investigation of hypersound magnetic and elastic oscillations excitation by use of alternating magnetic field in normal magnetized plate. Distinctive features of frequency division and multiplication are studied in the paper. The relation between processes of frequency division and multiplication and structure of the plate as well as parameters of saturation magnetization, anisotropy constant, magnetic and elastic damping has been revealed.
\end{abstract}

\section{Introduction}

The magnetoelastic oscillations generation caused by magnetostriction is one of the interesting interaction mechanism of magnetic, elastic and magnetoelastic subsystems, that attracted attention of researchers for a long time [1-5]. This process can be executed in case of action on the plate by alternating magnetic field, hypersound oscillations as well as pulsed magnetisation reversal in a constant field. Dimensions and shape of a plate are fitted in such a way that avoid chance of spin waves excitation [6]. The present work deals with investigation of the features of the magnetization vector nonlinear precession and the elastic displacement excited by alternating magnetic field. For this study, sample with Ni parameters has been taken which is also of technical interest.

\section{Geometry of the problem and basic equations}

The plane-parallel ferrite plate has thickness $d$. The external DC magnetic field $\mathbf{H}_{0}$ is applied perpendicularly to the plane of the plate as shown in Fig. 1. Right-hand circularly polarized alternating field is applied in the plate plane.

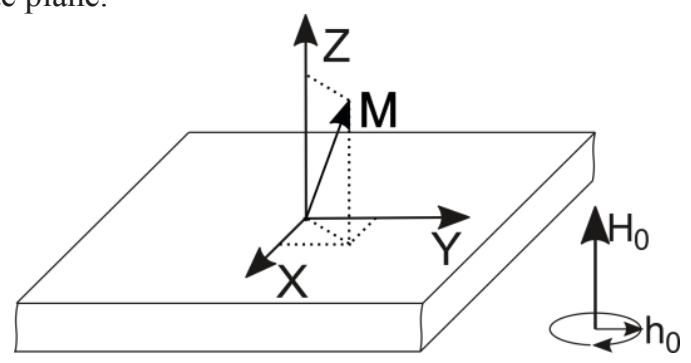

Fig. 1. Geometry of the problem.
The problem is solved in a Cartesian coordinate system Oxyz. The plane Oxy of the coordinate system coincides with the plane of the plate. The coordinate axes are parallel to the edges of the cube crystallographic cell. The center of the coordinate system $\mathrm{O}$ is in the center of the plate, so that the plate planes coordinates are $z= \pm d / 2$. We consider only excitement of the shear elastic oscillations.

Assuming that the total energy density of the plate $U$ in the field $\mathbf{H}=\left\{0 ; 0 ; \mathbf{H}_{0}\right\}$ equals the sum of magnetic, elastic and magnetoelastic energy densities, we obtain:

$$
\begin{aligned}
U= & -M_{0} h_{x} m_{x}-M_{0} h_{y} m_{y}-M_{0} H_{0} m_{z}+ \\
+ & 2 \pi M_{0}^{2} m_{z}^{2}+2 c_{44}\left(u_{x y}^{2}+u_{y z}^{2}+u_{z x}^{2}\right)+ \\
& +2 B_{2}\left(m_{x} m_{y} u_{x y}+m_{y} m z u_{y z}+m_{z} m_{x} u_{z x}\right)
\end{aligned}
$$

where $\mathbf{m}=\mathbf{M} / M_{0}$ - normalized magnetization vector, $M_{0}$ the saturation magnetization of the magnetic plate, $u_{\mathrm{ij}}-$ the components of the strain tensor, $c_{44}$ - the elastic constant and $B_{2}$ - the magnetoelastic coupling constant.

The initial equations for the system are the LandauLifshitz-Gilbert equation and the equations for the elastic displacement vector components $\mathrm{u}_{\mathrm{x}, \mathrm{y}}$ :

$$
\begin{aligned}
& \frac{\partial \vec{m}}{\partial t}=-\gamma\left[\vec{m} \times \vec{H}_{e}\right]+\alpha\left[\vec{m} \times \frac{\partial \vec{m}}{\partial t}\right], \\
& \frac{\partial^{2} u_{x, y}}{\partial t^{2}}=-2 \beta \frac{\partial u_{x, y}}{\partial t}+\frac{c_{44}}{\rho} \cdot \frac{\partial^{2} u_{x, y}}{\partial z^{2}},
\end{aligned}
$$

\footnotetext{
* Corresponding author: dpleshev@gmail.com
} 
where $\gamma$ - the gyromagnetic ratio; $\alpha, \beta$ - damping constants of the magnetic and elastic subsystems.

The effective field is determined by the following equation:

$$
\overrightarrow{\mathbf{H}}_{e}=-\frac{1}{M_{0}} \cdot \frac{\partial U}{\partial \overrightarrow{\mathbf{m}}}
$$

The boundary conditions are:

$$
\left.c_{44} \frac{\partial u_{x, y}}{\partial z}\right|_{z= \pm d / 2}=-B_{2} m_{x, y} m_{z} .
$$

The system of the equations [7] was solved numerically by the Runge-Kutta 7-8 orders method with control of the integration at every step length.

The material parameters used in the calculation: $\rho=8.968 \mathrm{~g} \cdot \mathrm{cm}^{-3}, \quad M_{0}=480 \mathrm{G}, \quad c_{44}=1.2 \cdot 10^{11} \mathrm{erg} \cdot \mathrm{cm}^{-3}$, $B_{2}=0.16 \cdot 10^{9} \mathrm{erg} \cdot \mathrm{cm}^{-3}, \alpha=0.035$.

\section{Frequency division}

Consider the case when the frequency of elastic resonance was multiple of alternating field frequencies, i.e. thickness of the plane was selected multiple of resonance thickness i.e. $d=n \cdot d_{\mathrm{r}}$, where $n$ is multiplicity, $d_{\mathrm{r}}$ - the resonance thickness.

The process of frequency division is followed by two transient regimes connected with relaxations of elastic and magnetic oscillations. Optimum conditions for frequency division can be traced when relaxation of magnetic oscillations finishes whereas relaxation of elastic oscillations does not appear. The second necessary condition is nonlinear regime of magnetoelastic oscillations when $\mathbf{h}_{0} \geq 10 \mathrm{Oe}$.

Development of magnetization oscillations and elastic displacements in the nonlinear $\left(\mathbf{h}_{0}=1000\right.$ Oe $)$ case can be seen in the Fig. 2 .
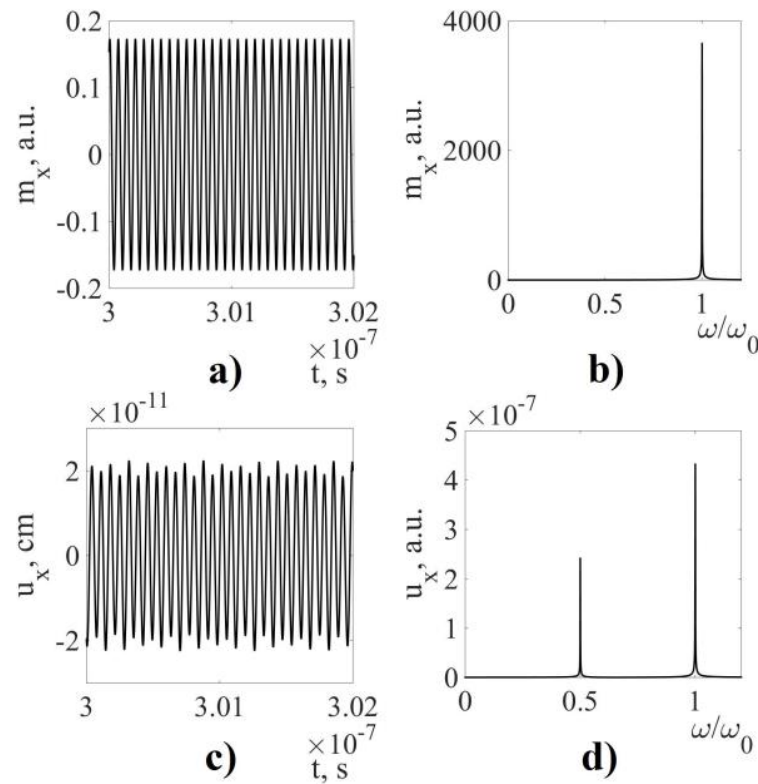

Fig. 2. Development of magnetization oscillations and elastic displacements (a, c) and their spectra (b, c) in case of $\mathbf{h}_{0}=1000$ Oe and $n=2$.
Fig.2b and Fig.2d illustrate that magnetization oscillations and elastic oscillations have a spectral peak on excitation frequency and elastic oscillations have a spectral peak on elastic resonance frequency of the plate in this case and the elastic resonance frequency occurs on one-half of excitation frequency. It should be noticed here, that the elastic displacement frequency is equal to excitation frequency after magnetic and elastic subsystems relaxation.

We consider the case integer frequency division when the elastic resonance frequency of the plate is a multiple of the frequency of the alternating field at a ratio of $1 / 2,1 / 3$ and $1 / 4$, which corresponds to $n=2,3$ and 4 correspondingly.

Fig.3 displays that elastic oscillation spectra have two peaks. The one is on the excitation frequency and the other on the frequency of elastic resonance. The magnetic oscillations hold the shape while the elastic oscillations have the shape distortion due to undesired oscillation that occur on excitations frequency.
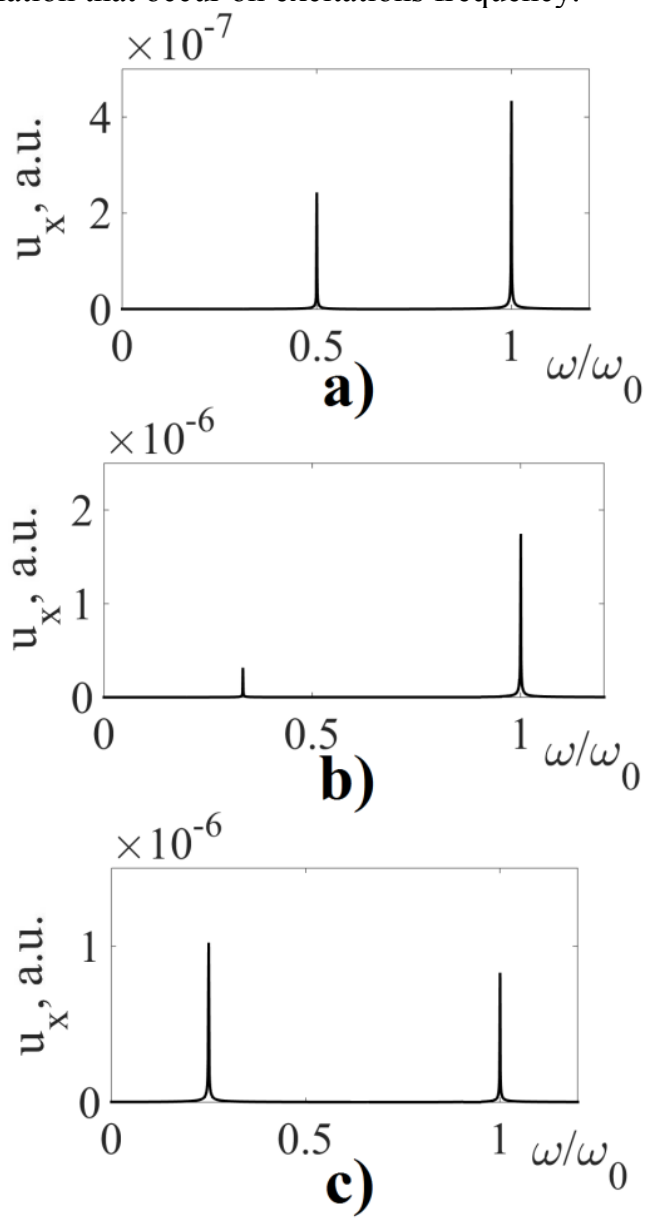

Fig. 3. Spectra of elastic displacements oscillations in case of $\mathbf{h}_{0}=1000$ Oe and (a) $n=2$, (b) $n=3$, (c) $n=4$.

In case of the fractional frequency division when $\mathrm{n}=2.2, \quad \mathrm{n}=2.5$ and $\mathrm{n}=2.8$, the spectra of elastic displacement get a strong change as is shown in Fig.4.

The paper [8] considers the situation where the multiplicity shifted to some integer, for example, for $\mathrm{n}=$ 2.2 or $\mathrm{n}=2.8$, the amplitude of magnetization oscillations on the frequency of the elastic resonance is two orders of magnitude higher if the division 
multiplicity corresponds to $\mathrm{n}=2.5,3.5$, i.e. multiplicity is in the middle of the neighboring integer multiplicities. As opposed to [8] in this case the similar dependence is not available.
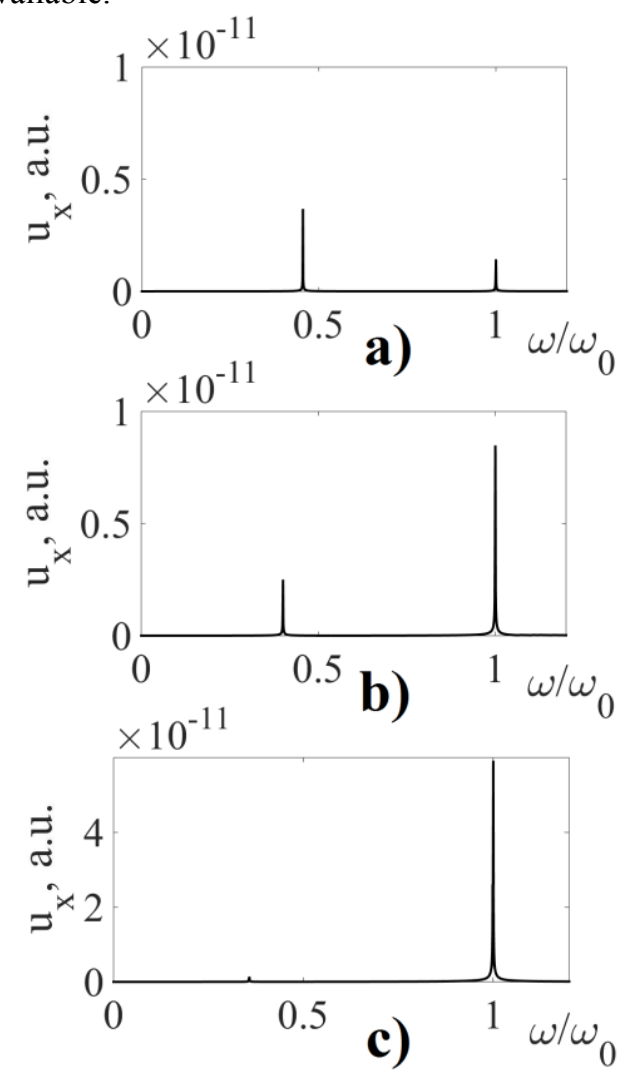

Fig. 4. Spectra of elastic displacements oscillations in case of $\mathbf{h}_{0}=1000$ Oe and (a) $n=2.2$, (b) $n=2.5$, (c) $n=2.8$.

The spectrum analysis of elastic oscillations makes it possible to determine the distribution of amplitudes maxima on excitation frequency and frequency of elastic resonance in relation to division multiplicity that are shown in Fig. 5.

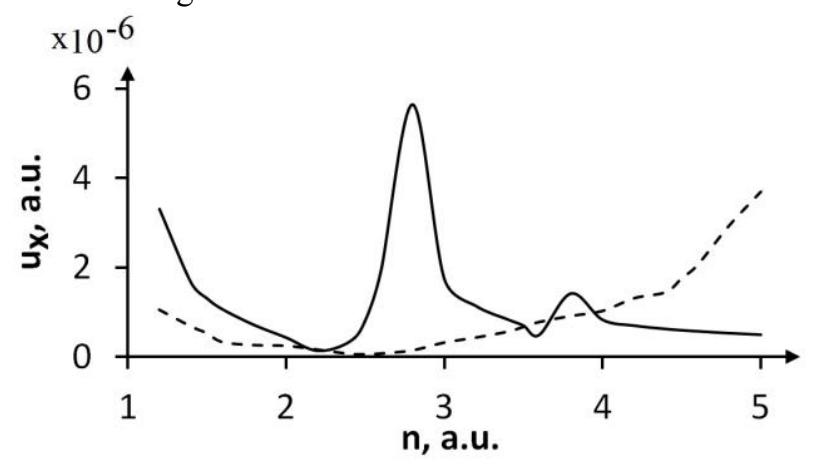

Fig. 5. Distribution of elastic oscillations amplitudes as a function of the division multiplicity on excitations frequency (solid line) and on the frequency of the elastic resonance (dash line).

Amplitudes of elastic oscillations decrease exponentially on frequency of excitation with increase of multiplicity and have peaks at multiplicity $n=2.8$ and $\mathrm{n}=3.8$. Note that the amplitudes of the magnetic subsystem are not prone to considerable changes.

The distribution of the maximum amplitudes as a function of the fission multiplicity has a shape of an irregular-shaped parabola with a vertex at $n=2.5$ on the frequency of the acoustic resonance. The efficient frequency division is possible in case of multiplicity value $n<1.5$ or $n>4$.

\section{Frequency multiplication}

Frequency multiplication is implemented by fitting of thickness of the plate in such a way that the frequency of elastic resonance was multiple bigger than excitation frequency. That is the thickness of the plate equals $d=$ $d_{\mathrm{r}} / n$, where $n$ is multiplicity, $d_{\mathrm{r}}$ - the resonance thickness. The optimal conditions of frequency multiplications are analogous to case of frequency division. The excitation field should be linearly polarized in order the frequency multiplication is efficient. The amplitudes of excitation field components are equal to $h_{\mathrm{x}} \neq 0, h_{\mathrm{y}}=0$.

Consider the case of the frequency multiplication when $n=2,3,4$, i.e. $n$ is integer. The spectra of elastic oscillations contain frequency component of elastic resonance and excitation frequency component as is shown in Fig. 6. The oscillation amplitudes ratio on the excitation and elastic resonance frequencies changes subject to increasing multiplicity of frequency multiplication.

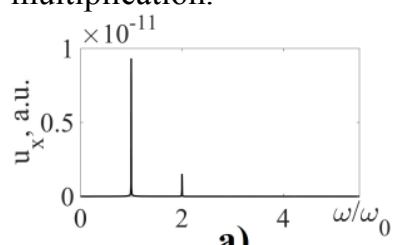

a)
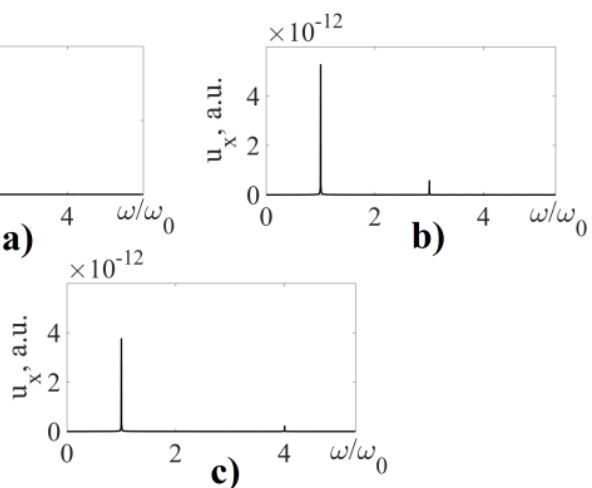

Fig. 6. Spectra of elastic displacements oscillations in case of $\mathbf{h}_{0}=1000$ Oe and (a) $n=2$, (b) $n=3$, (c) $n=4$.

Fig.7 displays spectra of elastic oscillations in case of fractional frequency multiplication when $n=2.2, n=2.5$ and 3.5 .
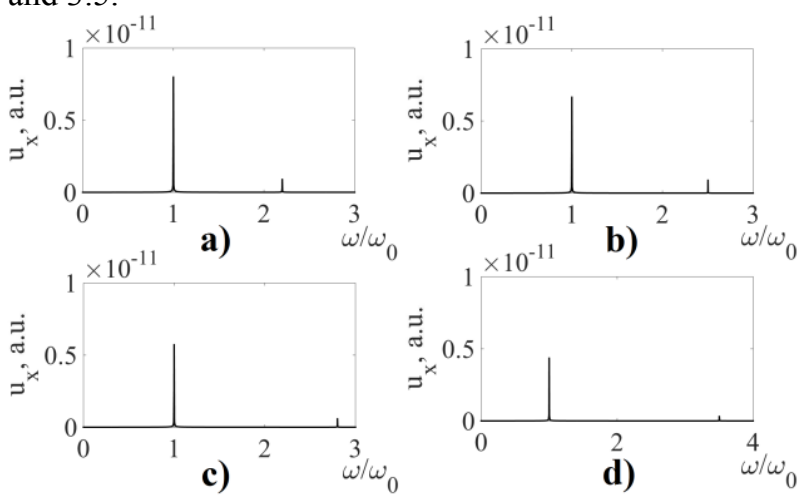

Fig. 7. Spectra of elastic displacements oscillations in case of $\mathbf{h}_{0}=1000$ Oe and (a) $n=2.2$, (b) $n=2.5$, (c) $n=2.8$, (d) $n=3.5$. 
It is important to note that magnitude of elastic oscillations on the frequency of elastic resonance decreases with increase of multiplicity. Considering the fact that the amplitude of elastic oscillations on frequency of elastic resonance is an order of magnitude less than the amplitude of elastic oscillations on excitation frequency, multiplication of frequency is possible, but it's of no practical interest at high multiplicity.

Fig. 8 depicts the amplitudes maxima distribution of elastic oscillations on excitation frequency and frequency of elastic resonance in relation to multiplicity of multiplication. Amplitudes of elastic oscillations decrease monotonically on the frequency of excitation and the frequency of elastic resonance with increase of multiplicity. The elastic oscillations on the frequency of elastic resonance have peaks at multiplicity $n=2.2$ and $n=2.5$. The efficiency of frequency multiplication decreases with increase of multiplication multiplicity.

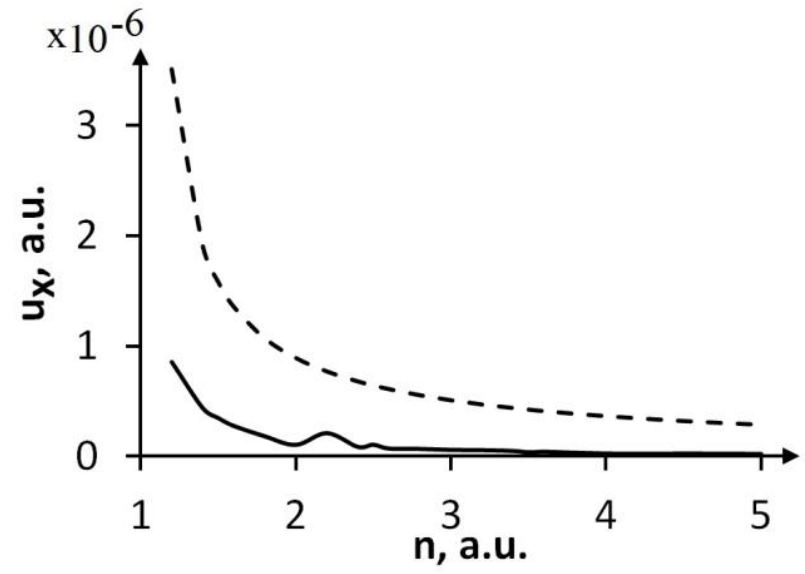

Fig. 8. Distribution of elastic oscillations amplitudes as a function of the multiplication multiplicity on excitations frequency (dash line) and on the frequency of the elastic resonance (solid line).

\section{Conclusion}

Thus the coupled oscillations of magnetization and elastic displacement in normally magnetized ferrite plate that possess magnetoelastic properties are considered in the paper.

The research shows that the efficient processes of frequency division and multiplication are possible only when regime of excitement is nonlinear. In case of multiplication, polarization of excitation alternating field should be linear.

Correlation of division and multiplication processes with time of magnetic and elastic relaxations are described. The paper reveals the fact that stable division and multiplication processes exist when relaxation time of elastic oscillations are bigger by an order of magnitude than relaxation time of magnetic oscillations.

The dependencies of effective division and multiplication conditions are defined. The spectrum analysis showed that components on exciting frequencies are dominant over components on multiply and division frequencies. However, in case of frequency division, components on frequency of elastic resonance are greater than components on exciting frequency if multiplicity is more than $n>4$.

This work was supported by RFBR (grant no. 17-0201138-a, grant 17-57-150001 НЦНИ-а.).

\section{References}

1. R.L. Comstock, R.C. LeCraw, Generation of microwave elastic vibrations in a disk by ferromagnetic resonance, J. Appl. Phys., 34 (1963), 3022.

2. M. Ye, H. Dötsch. Magnetoelastic instabilities in the ferrimagnetic resonance of magnetic garnet films, Phys. Rev. B, 44 (1991), 9458.

3. A.M. Shutyĭ, D.I. Sementsov. Nonlinear effects of magnetization precession near ferromagnetic resonance, Physics of the Solid State. 42 (2000) 1306-1309.

4. L. Dreher et al. Surface acoustic wave driven ferromagnetic resonance in nickel thin films: Theory and experiment, Phys. Rev. B, 86 (2012), 134415(13).

5. L. Thevenard et al. Irreversible magnetization switching using surface acoustic waves, Phys. Rev. B, 87 (2013), 144402(9).

6. V.S. Vlasov, L.N. Kotov, V.G. Shavrov, V.I. Shcheglov, Nonlinear excitation of hypersound in a ferrite plate under the ferromagnetic-resonance conditions, J. Comm. Tech. El. 54(2009) 821-832.

7. M.S. Kirushev, V.S. Vlasov, D.A. Pleshev, F.F. Asadullin, L.N. Kotov, V.G. Shavrov, V.I. Shcheglov, Solid State Phenomena Vols 233-234 (2015) 73-78.

8. F.F. Asadullin, S.M. Poleshikov, D.A. Pleshev, L.N. Kotov, V.S. Vlasov, V.G. Shavrov, V.I. Shcheglov, Journal of Siberian Federal University. Mathematics \& Physics 2017, 10(1), 36-39. 